\title{
Hypercomplex Factorization of the Helmholtz Equation
}

\section{K. GÜRLEBECK}

Hauptergebnis der Arbeit ist die Angabe einer globalen hyperkomplexen Faktorisierung des Dirichlet-Problems für die Helmholtz-Gleichung. Dieses Resultat gestattét die Zurückführung des betrachteten Randwertproblems auf zwei Randwertprobleme erster Ordnung für verallgemeinert analytische Funktionen.

Основным результатом работы является постросние гяобальной гипсрномплексной фанторизации задачи Дирихле для уравнения Гельмгльца. Тақим образом названная задача может. быть сведена к двум краевым задачам первого порлдка для обобщенио аналитических функцин.

The main result of this paper is the construction of a global hypercomplex factorization of the Dirichlet problem for, the Helmboltz equation. This result permits the reduction of the con. sidered boundary value problem into two boundary value problems of first order for generalized analytic functions.

\section{Introduction}

In the last years factorizing principles for the solution of elliptic boundary value problems have got an increasing importance. Using the concept of generalized analytic functions [1] ànd an operator calculus [7] global factorizations of such problems for the laplace equation and for the equations of linear elasticity were found in [4]. The aim of this paper is to state analogous results for the Helmholtz equation.

\section{Notations}

Let $Q$ be the quaternionic algebra with basic elements $e_{1}=(1,0,0,0), \ldots, e_{4}$ $=(0,0,0,1)$. Wach element $a \in Q$ has an 'unique representation $a=\sum_{i=1}^{4} a_{i} \cdot e_{i}$ with real coefficients $a_{i}$. The conjugate element $\bar{a}$ is defined by $\bar{a}=a_{1} e_{1}-\sum_{i=2}^{4} a_{i} e_{i}$ and a norm by $|a|=(a \circ \bar{a})^{1 / 2}$, where $\circ$ denotes the multiplication of quaternions. Tet $G \subset \mathbf{R}^{3}$ be a bounded domain with smooth boundary $\Gamma$. A quaternionic-valiued function $f$ will be written as

$$
f(x)=\sum_{i=1}^{4} f_{i}(x) e_{i} \text { with } f_{i}: G \rightarrow \mathbf{R}^{1}
$$

Define the differential operator $D(F)$ as $D(\nabla)=\sum_{i=2}^{4} e_{i} \frac{\partial}{\partial x_{i}}$, let the differential ope- 
rator $D\left(\nabla_{1}\right)_{r}$ be given by $D(\nabla)_{r}=\sum_{i=2}^{4} \frac{\partial^{*}}{\partial x_{i}} e_{i}$ and, finally, denote by $D_{\lambda}(V), D_{\lambda}{ }^{*}(\nabla)$, and $D_{\lambda}(\nabla)$, the operators $D_{\lambda}(V)=\lambda e_{1} \div \frac{1}{+} D(\nabla), D_{\lambda}^{*}(\nabla)=\lambda e_{1}-D(\nabla)$, and $D_{\lambda}(\nabla)_{r}=\lambda e_{1}$ $+D(\nabla)_{r}$ with a real number $\lambda$. The normalized outer normal to $\Gamma$ will be denoted by $n$. Elements $x \in \mathbf{k}^{3}$ will be written in the form $x=\left(x_{2}, x_{3}, x_{4}\right)$ to get simplicity in index-calculation. With every $x \in \mathbf{1}^{3}$ we associate a corresponding element $D(x)=\sum_{i=2}^{4} x_{i} e_{i} \in Q$.

\section{Fundamental solutions}

The action of $\left(\Delta+\lambda^{2}\right) I$ on quaternionic-valued function $f$ will be defined by action of $\left(\Delta+\lambda^{2}\right)$ in usual sense on each component of $f$. A formal factorization of this operator is given by

$$
\left(\Delta+\lambda^{2}\right) I=D_{\lambda}(\grave{V}) D_{\lambda}^{*}(\nabla)=D_{\lambda}^{*}(\nabla) D_{\lambda}(\nabla) .
$$

The knowledge of fundamental solutions of the operators $D_{\lambda}(\nabla)$ and $D_{\lambda}^{*}(\Gamma)$ is . necessary for defining suitable integral operators and for constructing complete function systems. For the sake of simplicity, all following investigations will be carried out for the operator $D_{\lambda}(\nabla)$ only.

Theorem 1: Let $x, y \leqslant \mathbf{R}^{3}, x \neq y$ and

Then

$$
F_{\lambda}(x)=\frac{\lambda \cos \lambda|x|}{|x|} e_{1}^{*}+\frac{D(x)}{|x|^{2}} \lambda \sin \lambda|x|+\frac{D(x)}{|x|^{3}} \cos \lambda|x|
$$

$$
D_{\lambda}\left(\nabla_{x}\right) F_{\lambda}(x-y)=F_{\lambda}(x-y) D_{\lambda}^{*}\left(\nabla_{x}\right)_{r}=-4 \pi \delta(x-y) .
$$

Proof: A simple computation using $D\left(\nabla_{x}\right) \frac{D(x-y)}{|x-y|^{3}}=-4 \pi \delta(x-y)$. (see [2])

\section{Operators}

Let $G \subset \mathbf{R}^{3}$ be a bounded domain with Ijapunov boundary, $f$ a sufficiently smooth function defined in $\dot{G}$ or on $\Gamma$ and let $F_{z}$ be given by (1). Define

$$
\begin{aligned}
& \left(T_{\lambda} f\right)(x)=-\frac{1}{4 \pi} \int_{G} F_{\lambda}(x-y) \circ f(y) d G_{y} ; \quad x \in \mathbf{R}^{3}, \\
& \left(\dot{S}_{\lambda} f\right)(x)=\frac{1}{4 \pi} \int_{\Gamma} F_{\lambda}(x-y) \circ D(n)_{\nu} \circ f(y) d \Gamma_{\nu}, \quad x \notin \Gamma, \\
& \left(S_{\lambda} f\right)(x)=\frac{1}{2 \pi} \int_{V} F_{\lambda}(x-y) \circ D(n)_{\nu} \circ f(y) d \Gamma_{\nu}, \quad x \in \Gamma .
\end{aligned}
$$

Remark 1: The integral operators $T_{\lambda}, \dot{S}_{\lambda}$, and $S_{\lambda}$ are defined in analogy to the complex 1 -operator, the Cauchy integral operator and singular Cauchy integral operator, where the integral $\dot{S}_{\lambda} f$ is to be understood in the Cauchy principal value sense. In the case $\lambda=0$ we get the operators investigated in [7]. 
Lem ma 1 : Let $G \subset \mathbf{R}^{3}$ be a bounded domain with Ljapunov boundary and $f \in C^{1}(G)$ in $\dot{C}(\bar{G})$. Then

$$
f(x)=\left(\dot{S}_{\lambda} f\right)(x)+\left(T_{\lambda} D_{\lambda}(\nabla) f\right)(x) \text { for any } x \in G .
$$

Proof: We have

$$
1
$$

$$
\begin{aligned}
\left(T_{\lambda} D_{\lambda}(V) f\right)(x) & =-\frac{1}{4 \pi} \int_{G} F_{\lambda}(x-y) \circ D_{\lambda}\left(\nabla_{y}\right) f(y) d G_{y} \\
& =\lim _{\varepsilon \rightarrow 0}\left(-\frac{1}{4 \pi} \int_{G_{\varepsilon}} F_{\lambda}(x-y) \circ D_{\lambda}\left(\nabla_{\nu}\right) f(y) d G_{y}\right)
\end{aligned}
$$

.with

$$
\begin{aligned}
& G_{\varepsilon}=G \backslash\{y:|x-y| \leqq \varepsilon\}=G \backslash K_{x}(\varepsilon), \cdot \Gamma_{\varepsilon}=\partial K_{x}(\varepsilon) \\
& -\frac{1}{4 \pi} \int_{G_{\varepsilon}} F_{\lambda}(x-y) \circ D_{\lambda}\left(\Gamma_{\nu}\right) f(y) d G_{v} \\
& =-\frac{1}{4 \pi} \int_{G_{\varepsilon}}\left(F_{\lambda}(x-y) D_{\lambda}^{*}\left(\nabla_{y}\right)_{r}\right) \circ f(y) d \sigma_{y} \\
& -\frac{1}{4 \pi} \int_{\Gamma} F_{i}(x-y) \circ D(n) \circ f(y) d \Gamma_{y}+\frac{1}{4 \pi^{\prime}} \int F_{\lambda}(x-y) \circ D(n) \circ f(y) d \Gamma_{y} .
\end{aligned}
$$

Froin this equation we obtain the desired result by using the forniula (see [7])

$$
\lim _{\varepsilon \rightarrow 0} \int_{G_{\varepsilon}}\left[\frac{D(x-y)}{|x-y|^{3}} D\left(\nabla_{x}\right)_{r}\right] \circ f(y) d G_{y}=-4 \pi f(x)
$$

Lem ma 2: Let $\gamma_{0}$ be the trace operator in the sense of [5]. Under the same hypotheses as in Lemma 1 one has

$$
f(x)=\left(\dot{S}_{\lambda} \gamma_{0} f\right)(x) \quad \forall f \in \operatorname{Ker} D_{\lambda}(\nabla)(G), \quad x \in G
$$

Proof: Application of $(6)^{\circ}$ to $f \in \operatorname{Ker} D_{\lambda}(\nabla)(G)$

\section{Lémma 3: Under the hypotheses of Lemma 1}

$$
\left(D_{\lambda}(\nabla) T_{\lambda} f\right)(x)=f(x) \text { for any } x \in G .
$$

Pronf: In analogy to the proof of Lemma 1

- Reinark 2: Formula (6) generalizes the well-known Borel-Ponpeiu representation of complex function theory, (7) is an analogue of the Cauchy integral formula. Corresponding results for $\lambda=0$ are proved in $[1,7]$.

Lemna.4: Let $f \in C^{\alpha}(\Gamma)$ and suppose the boundary $\Gamma$ of $G$ is a Ljapunov surface. Then

$$
\begin{aligned}
& \lim _{\substack{x \rightarrow x_{0} \in I \\
x \in G}}\left(S_{\lambda} f\right)(x)=\frac{1}{2}\left[\left(S_{\lambda} f\right)\left(x_{0}\right)+f\left(x_{0}\right)\right]=:\left(I_{\lambda} f\right)\left(x_{0}\right), \\
& \lim _{\substack{x \rightarrow x_{0} \in I \\
x \in \bar{C}}}\left(\dot{S}_{\lambda} f\right) \cdot(x)=\frac{1}{2}\left[\left(S_{\lambda} f\right)\left(x_{0}\right)-f\left(x_{0}\right)\right]=:-\left(Q_{\lambda} f\right)\left(x_{0}\right) \text { for any } x_{0} \in \Gamma .
\end{aligned}
$$


Proof: A simple computation making use of some properties of weakly singular integral operators shows thát

$$
\begin{aligned}
\lim _{\substack{x \rightarrow x_{0} \in \Gamma \\
x \in i}}\left(\dot{S_{2}} f\right)(x)= & \frac{1}{4 \pi} \int_{\Gamma}\left[\frac{\lambda \cos \lambda\left|x_{0}-y\right|}{\left|x_{0}-y\right|}+\frac{D\left(x_{0}-y\right)}{\left|x_{0}-y\right|^{2}} \lambda \sin \lambda\left|x_{0}-y\right|\right. \\
\therefore \quad & \left.+\frac{D\left(x_{0}-y\right)}{\left|\dot{x}_{0}-y\right|^{3}} \sum_{i=1}^{\infty}(-1)^{i} \frac{1}{(2 i) !}\left|x_{0}-y\right|^{2 i} \lambda^{2 i}\right]_{;} D(n) \circ f(y) d \Gamma_{\nu} \\
& +\lim _{\substack{x \rightarrow x_{0} \\
x \in G}} \frac{1}{4 \pi} \int_{\Gamma}^{-\frac{D(x-y)}{|x-y|^{3}} \circ D(\ddot{n}) \circ f(y) d \Gamma_{\nu} .}
\end{aligned}
$$

(11) combined with, the result for $\lambda=0$ contained in [7] gives the assertion of the ' lemma. Analogous arguments yield (10)

I emm a 5: Let $f \in C^{\alpha}\left(l^{\prime}\right), \Gamma$ the Ljapunow boundary of $G$. Then

$$
\begin{aligned}
& S_{\lambda}^{2} f=f \\
& \dot{S}_{\lambda} P_{\lambda} f=\dot{S}_{\lambda} f, \\
& P_{\lambda}{ }^{2} f=P_{\lambda} f .
\end{aligned}
$$

Proof: It is obvious that $S_{\lambda}{ }^{2} f=f$ iff $P_{\lambda}{ }^{2} f={ }_{\lambda} f$. Formula (14) can be verified by applying $(6)$ to the function $S_{\lambda} f \in \operatorname{Ke} r D_{\lambda}(P)(G)$. Using (14) and applying $(6)$ to $\dot{S}_{\lambda} f-\dot{S}_{\lambda} P_{\lambda} f$ we arrive at $(13)$

Remark 3: Note that the above results remain true also under weaker smoothness conditions for the function $f$.

The purpose of what follows is to investigate the continuity properties of the operators $S_{\lambda}, S_{\lambda}$ and $T_{\lambda}$. From the representation

$$
\left(T_{\lambda} f\right)(x)=\frac{1}{4 \pi} \int_{G}\left(D_{\lambda}(\nabla) \frac{\cos i|x-y|}{|x-y|}\right) \circ f(y) d G_{y}
$$

we get

$$
T_{\lambda} f \in H^{s_{i}+1}(G) \text { for } \quad i \in H^{s}(G) .
$$

Hence,

$$
T_{\lambda} D_{\lambda}(\dot{\nabla}) f \in H^{s}(G) \text { for } f \dot{\epsilon} H^{s}(G) .
$$

From : (16) and (17) it follows that $\dot{S}_{\lambda} \gamma_{0} f \in H^{s}(G)$ for $f \in H^{s}(G)$. Well-known theorenis about traces together with formula (6) iniply that $S_{\lambda} \gamma_{0} f \in H^{8-1 / 2}(\Gamma)$ and $P_{\lambda} \gamma_{0} f$ $\in H^{8-1 / 2}(\dot{\Gamma})$ for $f \in H^{8}(G)$. It can be shown that ' $S_{\lambda}, P_{\lambda} \in L\left(H^{s}(\Gamma)\right)$.

Lèmma 6: Let $G$ be a bounded domain with Ljapunov boundury $\Gamma$ and $s>3 / 2$. Then

$$
\dot{S}_{\lambda} \in L\left(H^{s-1 / 2}(\Gamma), H^{s}(G) \cap \operatorname{Ker} J_{\lambda}(\nabla)(G)\right) \text {. }
$$

Proof: It will be shown that

$$
\left(H^{s}(G), H^{8-1}(G), H^{s-1 / 2}(\Gamma), D_{\lambda}(\nabla), \gamma_{0}\right) .
$$


is a Sard system with completeness condition (for the definition of Sard systems see $[3,6])$. We have

$$
\begin{aligned}
\because \quad & D_{\lambda}(\nabla) \in L\left(H^{s}(G), H^{s-1}(G)\right) \\
& \operatorname{im} D_{\lambda}(\nabla)=\overline{\operatorname{im} D_{\lambda}(\nabla)}=H^{s-1}(G) \cdot(\text { see }(8) \text { and }(16)) \\
& \gamma_{0} \in L\left(H^{s}(G), H^{s-1 / 2}(\Gamma)\right) \text { and im } \gamma_{0}=H^{s-1 / 2}(\Gamma)[5] \\
& H^{s}(G) \cap \operatorname{Ker} D_{\lambda}(\nabla)(G) \cap \operatorname{Ker} \gamma_{0}=\{0\} \quad(\text { see }(12)) \\
& \gamma_{0}\left(\operatorname{Ker} D_{\lambda}(\nabla)(G) \cap H^{s}(G)\right)=\overline{\gamma_{0}\left(\operatorname{Ker} D_{\lambda}(\nabla)(G) \cap H^{s}(G)\right)}
\end{aligned}
$$

To prove (18) we take a sequence

$$
\left\{y_{n}\right\}_{n=1}^{\infty} \subset \dot{H^{s-1 / 2}}(\Gamma) \cap \gamma_{0}\left(\operatorname{Ker} D_{\lambda}(\nabla)(G)\right)
$$

with $y_{n} \underset{n \rightarrow \infty}{\longrightarrow} y_{0}$ in $H^{s-1 / 2}(\Gamma)$. Then one has:

$$
\begin{array}{r}
S_{\lambda} y_{n}=y_{n} \Rightarrow S_{\lambda} y_{0}=y_{0} \Rightarrow P_{\lambda} y_{0}=y_{0} \\
\Rightarrow \dot{S}_{\lambda} y_{0} \in \operatorname{Ker} D_{\lambda}(\nabla)(G) \cap H^{s}(G)
\end{array}
$$

and

$$
y_{0}=\gamma_{0} \dot{S}_{\lambda} y_{0} \in \gamma_{0}\left(\operatorname{Ker} D_{\lambda}(\nabla)(G)\right) \cap \dot{H}^{s-1 / 2}(\Gamma)
$$

From [3: Lemma 4] and from what has just been proved we obtain the estimate

$$
C_{1} \hat{\|} f\left\|_{H^{*}(G)} \leqq\left(\left\|D_{\lambda}(\nabla) f\right\|_{H^{i-1}(G)}^{2}+\left\|\gamma_{0} f\right\|_{H^{8-1 / 2(r)}}^{2}\right)^{1 / 2} \leqq C_{2}\right\| f \|_{H^{2}(G)}
$$

or any $f . \in H^{s}(G)$. with $0<C_{1}, C_{2}<\infty$ independent of $f$. For $f \in \operatorname{Ker} D_{\lambda}(D)(G)$. $\cap H^{s}(G)$ the inequality, (19) implies that

$$
C_{1}\|f\|_{H^{\prime}(G)} \leqq\left\|\gamma_{0} f\right\|_{H^{s-1 / 2}(\Gamma)} \leqq C_{2}\|f\|_{H^{*}(G)} \bullet
$$

From (20) and the continuity of $P_{\lambda}$ in $H^{s-1 / 2}(\Gamma)$ the statement of the lemima follows

\section{Factorization}

In the following we shall show the main result of this paper, namely, that the Dirichlet problem for the Helmholtz equation permits a global factorization into two boundary value problems of first order. '

- Lemma 7! Let $G \subset \mathbf{R}^{3}$ be a bounded domain, $\Gamma=\partial G$ a Ljupunov surface, and $s>3 / 2$. Then the boundary value problem

$$
\begin{aligned}
& D_{\lambda}(D .) u=0 \text { in } G, \\
& \gamma_{0} u=g \text { on } I^{\top}
\end{aligned}
$$

- has a solution $u \in H^{s}(G)$ iff $g \in H^{s-1 / 2}(\Gamma) \cap \operatorname{im} P_{\lambda}$.

Proof: 1. Let $u \in H^{s}(G)$ be a solution of (21). From (7) and (9) then results that $\gamma_{0} u=P_{\lambda} \gamma_{0} u$.

2. Let $g \in H^{s-1 / 2}(\Gamma) \cap \operatorname{im} P_{\lambda}$. Then $(6)$, (7), (9), and Lemma 6 imply that $u=\dot{S}_{\lambda} g$. $\epsilon \operatorname{Ker} D_{\lambda}(\nabla)(G) \cap H^{8}(G)$ is a solution of the problem (21)

Remark 4: The solution of (21), if it exists, is unique. 
Theorem 2: Suppose $\hat{\imath}^{2}>0$ is not an eigenvalue of the $\Delta$-operator, $G$ is a bounded domain with Ljapunov boundary $\Gamma=\partial G, s>3 / 2$. Then the boundary value problem

$$
\begin{aligned}
& \Delta u+\dot{\imath}^{2} u=0 \quad \text { in } G, \\
& \gamma_{0} u=\dot{g} \text { on } \Gamma
\end{aligned}
$$

admits a factorization into two boundary value problems of first order

$$
\begin{aligned}
& D_{\lambda}(\nabla)=0 . \text { in } G, \quad D_{i}^{*}(\nabla) w=0 \text { in } G \text {, } \\
& \gamma_{0} v=P_{\lambda} g \text { on } \Gamma, \quad \gamma_{0} T_{\lambda} w=Q_{\lambda} g \text { on } \Gamma \text {. }
\end{aligned}
$$

and the solution $u$ of (22) is given by $u=v+T_{\lambda} w$.

Prof : 'The existence of a solution of the problem (23) has been shown in Lemma 7. From the solvability of the problem

$$
\begin{aligned}
& \Delta z+i^{2} z=0 \text { in } G, \\
& \gamma_{0} z=Q_{2} g: \text { on } \Gamma
\end{aligned}
$$

and from (6) we deduce that

$$
z=\stackrel{\circ}{S}_{\lambda} \gamma_{0} z \cdot+{ }^{\top} T_{\lambda} D_{\lambda}(\nabla) \ddot{z}=\stackrel{\leftrightarrow}{S}_{\lambda} P_{\lambda} Q_{\lambda} \gamma_{0} z+T_{\lambda}\left[D_{\lambda}(\nabla) z\right]=T_{\lambda} w .
$$

Because $z \in \operatorname{Ker}\left(\Delta+\lambda^{2}\right)(G)$, we get $\dot{w}=D_{\lambda}(\nabla) z \in \operatorname{Ker} D_{\lambda}^{*}(\nabla)(G)$

Remark 5: Corresponding results for the case $\lambda=0$ are contained in [7]. In [2], GoLDSCHMIDT considered factorization problems for the equation $\left(\Delta-\lambda^{2}\right) u=0$, $\lambda \in \mathbf{R}$.

\section{Complete function systems}

For the application of a numerical method based upon the result of Theorem 2 , it is necessary, to construct complete function systems in $\operatorname{Ker} D_{\lambda}(\nabla)(G) \cap H^{s}(G)$. For the sake of brevity the result will be given without proof.

Theorem 3: Let $G$ and $G_{A}$ be bounded domains with Lgapunov boundaries $\partial G=\Gamma$ and $\partial G_{A}=\Gamma_{A}$ such that $\bar{G} \subset G_{A}$. Further let $\left\{x_{i}\right\}_{i-1}^{\circ}$ be a dense subset of $I_{A}$ and the functions $\varphi_{i}(x)=H_{\lambda}\left(x-x_{i}\right)$ be defined as in Section 3. Then the system $\left\{\varphi_{i}\right\}_{i-1}^{\infty}$ $\subset L_{2}(G) \cap \operatorname{Ker} D_{\lambda}(\nabla)(G)$ is conplete in $L_{2}(G) \cap \operatorname{Ker} D_{2}(\nabla)(G)$.

Remark 6: Starting from this theorem, one can show the completeness of the system $\left\{\varphi_{i}\right\}_{i=1}^{\infty}$ in $H^{s}(G) \cap \operatorname{Ker} D_{\lambda}(V)(G)$ and then, using theorems about traces, its completeness in $H^{\varepsilon}(\Gamma) \cap \operatorname{im} P_{\lambda}$.

Remark 7: Using the systeni $\left\{\varphi_{i}\right\}_{i-1}^{\infty}$, function, systems with better numerical properties can be constructed with the method denonstrated in [4].

\section{REFERENCES}

[1] Brackx, F., Delangee, F.; and F. Sommen: Clifford analysis (Research Notes in Mathematics 76). London: Pitman 1982.

[2] Goldschmidt, B.: Verallgemeinert analytische Vektoren im . $\mathbf{R}^{n}$. Dissertation B. Halle: Martin-Luther-Universität Halle-Wittenberg 1980. 
[3] GürLEBECK; K.: Zur optimalen Interpolation verallgemeinert analytischer Funktionen. Wiss. Z. Tèchn. Hochschule Karl-Marx-Stadt 25 (1983), 324-325 (Depotinformation).

[4] GürLebecK, K.: Uber die optimale Interpolation verallgemeinert analytischer quaternionenwertiger Funktionen und ihre Anwendung zur näherungsweisen Lösung wichtiger räumlicher Randwertaufgaben der Mathematischen Physik. Dissertation. Kárl-MarxStadt: Techn. Hochschule 1984.

[5] Лионс, Ж.-Л., и Э. МАджЕнЕс: Неоднородные граничные задачи и их прило́жения. Москва: Јзд-во Мир 1971.

[6] SARD, A.: Approximation Based on Nonscalar Observations. J. Approx. Theory 8 (1973), 1. 315-334.

[7] SpRöśsig, W.: Ưber eine mehrdimensionale Operatorrechnung mit Operatoren über beschränkten Gebieten des Enklidischen Raumes und deren Anwendung zúr Lösung partieller Differentialgleichungen. Dissertation B. Karl-Marx-Stadt: Techn. Hochschule.1979.

Mạnuskripteingang: 14.12.1984

VERFASSER :

Dr. Klaus Gürlebeck

Sektion Mathematik'der Technischen Hochśchule

.DDR-9010 Karl-Marx-Stadt, PSF 964 